\title{
Deoxidation Impact on Non-Metallic Inclusions and Characterization Methods
}

\author{
Cécile Nicoli $^{\mathrm{a}, \mathrm{b}, \mathrm{c} *}$, Jean-François Carton ${ }^{\mathrm{b}}$, Alexis Vaucheret ${ }^{\mathrm{a}, \mathrm{c}}$, Philippe Jacquet ${ }^{\mathrm{a}, \mathrm{c}}$ \\ ${ }^{a}$ LaBoMaP, Arts et Métiers ParisTech, 71250 Cluny, France \\ ${ }^{\text {b }}$ CastMetal, 1 Boulevard de la Boissonnette, 42110 Feurs, France \\ ${ }^{c}$ Université de Lyon, ECAM Lyon, INSA-Lyon, LabECAM, F-69005 Lyon, France \\ *e-mail: cecile.nicoli@ecam.fr
}

Received: 22 September 2017/Accepted: 13 December 2017/Published online: 31 January 2018

This article is published with open access at AGH University of Science and Technology

\begin{abstract}
Deoxidation is an unavoidable step in the elaboration of steel. The study of its influence could improve the quality of low-carbon steel (0.20-0.25 wt.\% of carbon). There are many deoxidation methods, and the most-common one consists of adding aluminum. Although it is a classic method, determining the optimal process parameters (quantity, yield, etc.) could be very sensitive. Deoxidation plays a determining role on inclusion cleanliness, especially on sulfide morphology. In order to control the efficiency of deoxidation, different techniques can be used. In this paper, an automated counting procedure on a scanning electron microscope with a field emission gun (FEG-SEM) is presented. This method was applied on samples cast in our laboratory under different deoxidation conditions. According to this, the resulting inclusion population is correlated with the aluminum content to find the optimal process parameters.
\end{abstract}

\section{Keywords:}

sulfide morphology, deoxidation, non-metallic inclusions, low carbon steel, SEM- FEG

\section{INTRODUCTION}

One constant improvement taking place in metallurgy is steel cleanliness. During steelmaking after melting, one important step is deoxidation. This step is accomplished by adding elements that react with oxygen to form oxides [1]. These will migrate into the slag casted. Several alloys can be used for deoxidation [2]. In this study, the aim is to deoxidize as simply as possible and still have good steel cleanliness. The choice was made to add aluminum at different steps of the process. Aluminum is already being used in factories as the only deoxidizer [3]. The addition of aluminum will form $\mathrm{Al}_{2} \mathrm{O}_{3}$, which can create solid inclusions that are deleterious for steel mechanical properties such as impact toughness and fatigue. Aluminum also has an impact on the shape of the MnS inclusions [4-6]. The aluminum residual content should not fall below 0.015 wt. $\%$ to prevent the apparition of Type II MnS - the worst type for mechanical properties [6, 7].

There are three types of sulfide inclusions [8]. Type I is spherical, gray, and randomly distributed, and it is the least-deleterious of the three types. Type II is elongated, gray or yellowish, and aligned more or less discontinuously; this is the most-deleterious for steel. Type III is polyhedral, gray, and located at the triple seals of the solidification grains. Examples of sulfide inclusions are shown in Figure 1. a)

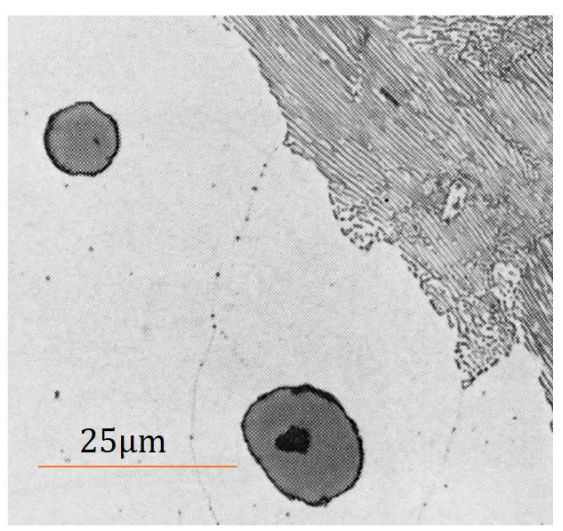

b)

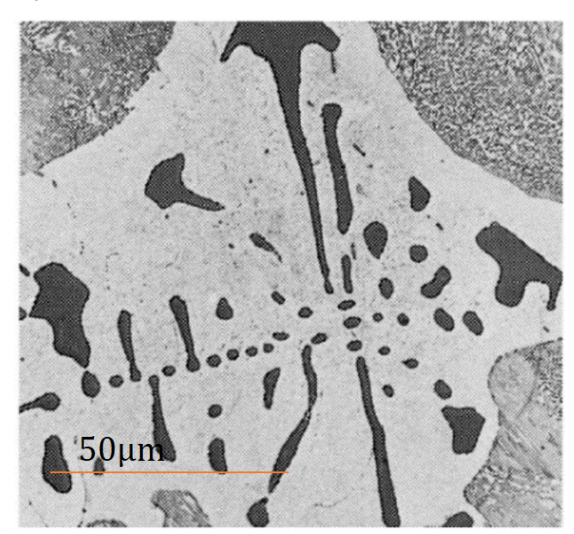

c)

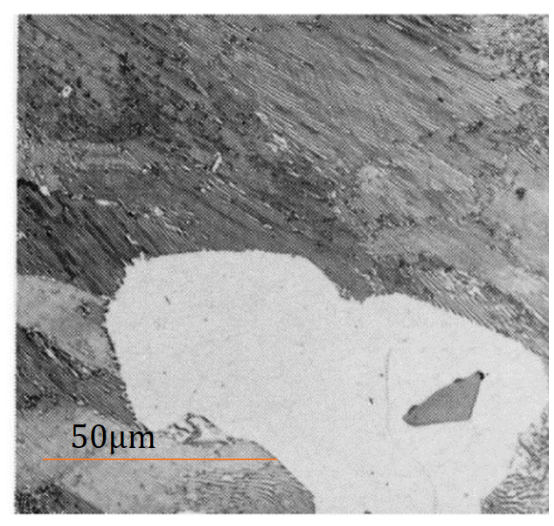

Fig. 1. Example of sulfur inclusion: a) Type I, $\times 800$; b) Type II, $\times 500$; c) Type III, $\times 500$ [9] 
There are also oxide inclusions; these are round and black not at all easy to see with an optical microscope. Other types of inclusions exist, but they are not found in this grade of steel.

The study of deoxidation will be done through a chemical analysis and counting. The use of a spectrometer and SEM-FEG will allow us to observe its effects. In order to do this, a casting protocol must be established, and a comparison with existing methods is necessary.

\section{EXPERIMENTS}

\subsection{Steel elaboration}

The specification of the steel used is G20 Mn5 (AFNOR) or 1.6220 (AISI) and its composition is described in Table 1.

Table 1

Specification of steel used in this study

\begin{tabular}{ccccccccc}
\hline $\mathbf{C}$ & $\mathbf{S i}$ & $\mathbf{M n}$ & $\mathbf{P}$ & $\mathbf{S}$ & $\mathbf{C r}$ & $\mathbf{M o}$ & $\mathbf{N i}$ & $\mathbf{A l}$ \\
\hline $0.17-$ & $<0.4$ & $1.00-$ & 0.035 & 0.035 & $<0.40$ & $<0.10$ & $<0.40$ & $<0.020$ \\
0.23 & & 1.50 & & & & & & \\
\hline
\end{tabular}

During this work, ingots are poured in green sand molds in our laboratory. The melting takes place in a medium-frequency induction furnace with a maximum power of $100 \mathrm{~kW}$ and capacity of 10 liters. The volume of the castings is 2.1 liters: sufficiently small to remain on the laboratory scale but sufficiently large to maintain a certain representativeness of the industrial parts. The ingot was previously designed by a numerical simulation thanks to the QuikCAST software: it takes 10 secs to fill the mold and about an hour to solidify. The ingot's dimensions are presented in Figure 2.

All cast ingots are analyzed in the laboratory via two main characterization techniques: spark spectrometry (for their chemical composition) and FEG microscopy (for the quantification of the inclusion population). This method will be detailed in Paragraph 2.2.

In order to demonstrate the influence of deoxidation on the inclusion property, it was necessary to set up a casting protocol to control and vary the deoxidation of the steel. For this, two types of deoxidation were carried out in this study:

- According to industrial processes that currently use $1 \mathrm{~kg}$ of Al per ton of steel, some aluminum was added directly inside the furnace to kill the bath.

- Additional varying quantities of aluminum were added inside the ladle to play a role in the microstructure.

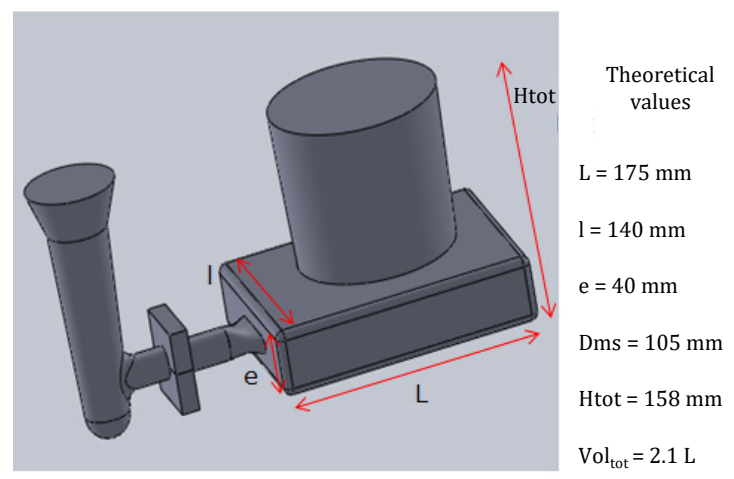

Fig. 2. Ingot dimensions

Two meltings were made in this study; the protocol of the aluminum addition is described in Table 2. For Melting 1, aluminum was added only in the ladle, so Step 2 from Figure 3 is skipped. For the first pouring, aluminum was placed at the bottom of the ladle, and for the second and third pouring, it was added in the middle of the ladle.

Table 2

Method to Al addition in steel

\begin{tabular}{ccccccc}
\hline & \multicolumn{2}{c}{ Melting 1 } & & \multicolumn{2}{c}{ Melting 2 } \\
\hline \multirow{2}{*}{ Test } & \multicolumn{2}{c}{ Al addition } & & \multicolumn{2}{c}{ Al addition } \\
\cline { 2 - 3 } \cline { 6 - 7 } & $\begin{array}{c}\text { Furnace, } \\
\text { g }\end{array}$ & $\begin{array}{c}\text { Ladle, } \\
\text { g }\end{array}$ & & $\begin{array}{c}\text { Furnace, } \\
\mathbf{g}\end{array}$ & $\begin{array}{c}\text { Ladle, } \\
\mathbf{g}\end{array}$ \\
\hline 1 & 0 & Bottom 8.7 & 4 & 60 & 0 \\
\hline 2 & 0 & Middle 7.0 & 5 & 60 & Middle 32 \\
\hline 3 & 0 & Middle 7.3 & 6 & 60 & Middle 17.5 \\
\hline- & - & - & 7 & 60 & Middle 8.4 \\
\hline
\end{tabular}

\begin{tabular}{|l|l|l|}
\hline $\begin{array}{l}\text { Step 1: before heating } \\
\text { loading the 10-liters } \\
\text { furnace }\end{array}$ & $\begin{array}{l}\text { Step 2: aluminium } \\
\text { addition }\end{array}$ & $\begin{array}{l}\text { Step 3: pouring melt steel } \\
\text { in ladle and Al addition }\end{array}$ \\
\hline
\end{tabular}

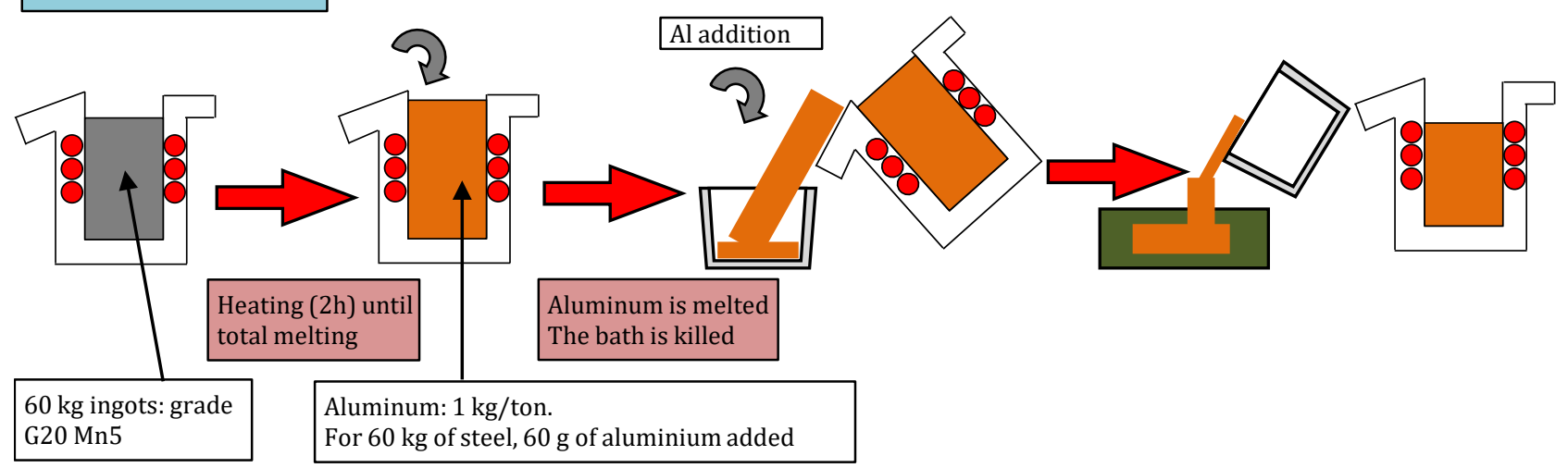

Fig. 3. Foundry process with specific deoxidation 


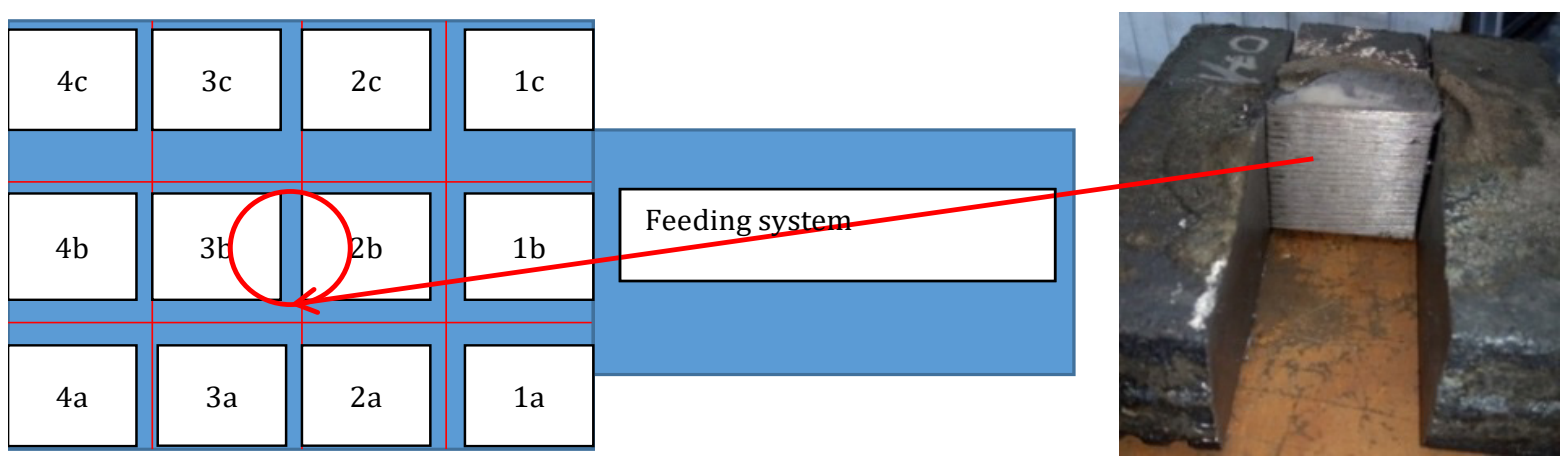

Fig. 4. Ingot cutting for analysis

For Melting 2, aluminum was added in the furnace (Step 2, Fig. 3) and a second addition was made in the ladle for Tests 5, 6, and 7. For Test 4, no aluminum was added in the ladle, so part of Step 3 was skipped.

The ingots are cut, and a sample is withdrawn in the center as shown in Figure 4. SEM analyses are made in the center of the ingot on a surface of $11 \mathrm{~mm}^{2}$ - the same surface observed in other competing software [10] for these kinds of analyses.

\subsection{Characterization methods}

To analyze the ingots' chemical compositions, spark spectrometer HORIBA has been used. In order to define the steel cleanliness, a method has been defined many years ago and is described in the standard NF EN 10247 [8]. This consists in counting inclusions on 20 random fields with $\times 500$ magnification. All types of inclusions have then been classified in a table, and the coefficients have been calculated to define the steel cleanliness. One drawback of this method is that some inclusions may be missed because the observer will not have distinguished them from the porosity.

In our study, the identification and classification of the inclusions has been realized automatically on a JEOL SEM-FEG (Scanning Electron Microscopy - Field Emission Gun). Image analysis software AZTEC (developed by Oxford) identified the different shades of gray of a sample to define the morphology of the inclusion and give its chemical composition by an EDS analysis. The chosen magnification is $\times 500$ (the same as the optical method), referring to the NF EN 10247 norm [8, 11, 12]. For this, a meticulous metallographic preparation is obligatory. The camera movement and EDS analyses are automated. The scanned fields are saved for possible future review.

The first step of this analyze is the acquisition of the first field (Fig. 5). The size of the field corresponds to the size of the optical method. The gray levels are defined in such a way as to detect a maximum number of particles (no matter their type). The studied area (as defined in Figure 4) has to be representative of the total sample in order to detect all types of inclusions.

The second step is automation. A large surface is defined by the operator; the software divides this large area into elementary fields (the same area as for the optical methods) and detects and analyzes each particle inside each elementary field. The chosen surface is determined in the software, and on each field, particles are detected and then analyzed. Several surfaces can be examined (as shown in Figure 6). This means that different samples can be analyzed during the automation. In Figure 6, we can see one surface whose automation is done and another surface whose analysis is still in progress; each light gray (green) part corresponds to one field. The size of the field is the same as that presented in Figure 5.

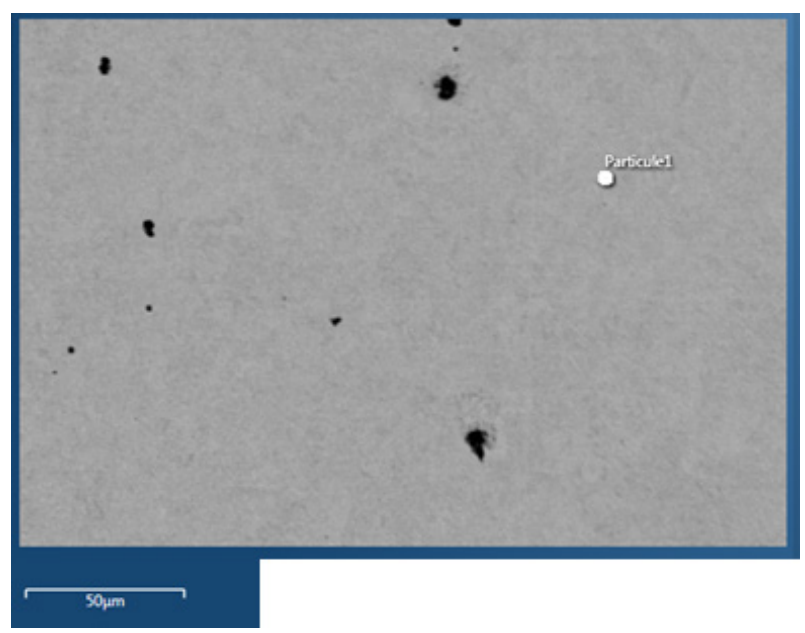

Fig. 5. Field and detection of particles in AZTEC software

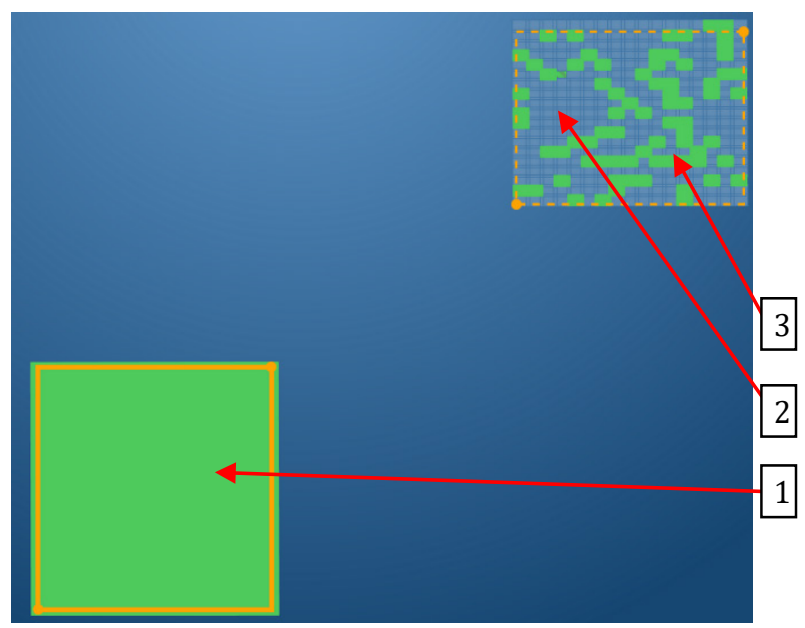

Fig. 6. Analyses in progress on AZTEC software: 1- area completely analyzed; 2 - studied area in progress; 3 - elementary fields already analyzed 
The last step is classifying the particles thanks to chemical analyses. The whole defined area chosen by the operator can be analyzed, or only a part of it (randomly selected by software). The method of classifying the particles is done according to their chemical composition and morphology. The classification of the morphology is made thanks to determining the threshold of the ratio of the equivalent diameters. It is comparable to the classification used in competing software [10]. This classification will apply to all of the particles after analysis and is the same for each surface evaluated. The software also identifies the shape of the inclusions, so a classification of the different types of sulfide inclusions is made [8].

As the system is automated, the time-saving is enormous when compared to conventional observation under an optical microscope. The conventional method is based on the counting of inclusions on 20 random fields of the same size as in Figure 5. The covered area is then less wide than with the software, and the accuracy for detecting the inclusions is subjective to the observer. For the same amount of time it takes to count the inclusions in 20 fields in the optical method, around 80 fields will be analyzed with the software; and more, the chemical composition of the inclusions will be determined, and shape measurement will be done. Another advantage is the easier way of distinguishing the porosity from the inclusions (thanks to the chemical composition).

\section{RESULTS}

\subsection{Aluminum addition}

The aluminum addition is based on the proportion used in the industry, which means $1 \mathrm{~kg} /$ ton of steel.

For Melting 1, aluminum was added only in the ladle. For the first pouring (1), aluminum was added at the bottom of the ladle, and for the second (2) and third (3) pouring, aluminum was added in the middle of the ladle. If it refers to Figure 3 , Step 2 is skipped. No aluminum is added in the furnace.

In the second melting, aluminum is added in the furnace and in the ladle. All steps from Figure 3 are realized.

Table 3 shows the residual amount of aluminum in the steel. Test 1 (aluminum added in the bottom of ladle) shows that it is better to add aluminum in the middle of the ladle. Furthermore, the carbon content is far from the expected value (0.31 wt.\%).
In the case of a unique addition in the furnace (Test 4), no residual aluminum is detected, as it was totally consumed. Only the aluminum added in the ladle appears in the residual amount.

The aluminum's addition in the furnace is necessary for killing the bath. It also reduces the activity in the oxygen, which can create defects like porosity in the steel. The aluminum completely reacts with the oxygen in the furnace. This can explain the very low residual amount of aluminum in the steel in Test 4.

Figure 7 shows that residual aluminum is proportional to aluminum added in the ladle. The aluminum is well-integrated; so, the oxygen activity must be very low, and the process of pouring steel from ladle to mold is not enough to generate a renewal of oxygen activity.

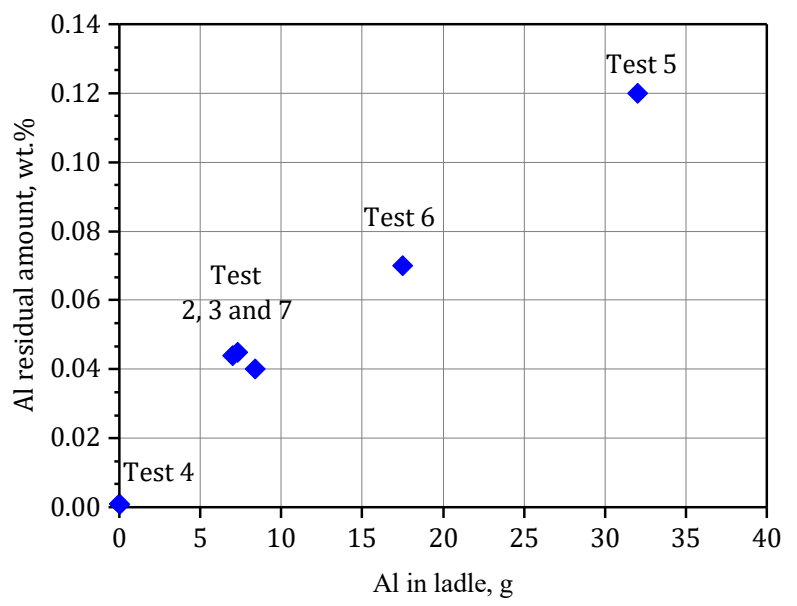

Fig. 7. Aluminum: residual amount (wt.\%) as a function of quantity added, $\mathrm{g}$

\subsection{Impact of aluminum deoxidtion on steel cleanliness}

SEM/FEG analyses were made on three types of deoxidation (as seen in Table 2):

- Aluminum added in furnace, Test 4 ,

- Aluminum added in ladle, Tests 2 and 3,

- Aluminum added in both, Tests 5, 6, and 7.

Then, these particles are classified and reported in Table 4.

Table 3

Spectrometer analyzes of different ingots

\begin{tabular}{|c|c|c|c|c|c|c|c|c|c|c|c|c|}
\hline- & Test & $\mathrm{C}$ & Mn & $\mathrm{Si}$ & $S$ & $\mathbf{P}$ & $\mathrm{Ni}$ & $\mathrm{Cr}$ & Mo & $\mathbf{V}$ & Al & $\mathrm{Cu}$ \\
\hline \multirow{3}{*}{ Melting 1} & 1 & 0.31 & 0.57 & 0.24 & 0.017 & 0.02 & 0.02 & 0.11 & 0.01 & 0.01 & 0.027 & 0.03 \\
\hline & 2 & 0.26 & 0.54 & 0.22 & 0.016 & 0.017 & 0.02 & 0.11 & 0.01 & 0.01 & 0.044 & 0.03 \\
\hline & 3 & 0.23 & 0.49 & 0.19 & 0.015 & 0.016 & 0.02 & 0.10 & 0.01 & 0.01 & 0.045 & 0.03 \\
\hline \multirow{4}{*}{ Melting 2} & 4 & 0.20 & 0.36 & 0.07 & 0.009 & 0.015 & 0.02 & 0.10 & 0.01 & 0.01 & 0.001 & 0.03 \\
\hline & 5 & 0.23 & 0.41 & 0.11 & 0.013 & 0.017 & 0.02 & 0.10 & 0.01 & 0.01 & 0.120 & 0.03 \\
\hline & 6 & 0.22 & 0.45 & 0.12 & 0.012 & 0.017 & 0.02 & 0.10 & 0.01 & 0.01 & 0.070 & 0.03 \\
\hline & 7 & 0.22 & 0.45 & 0.12 & 0.011 & 0.017 & 0.02 & 0.10 & 0.01 & 0.01 & 0.040 & 0.03 \\
\hline
\end{tabular}


Table 4

Inclusions analyses (SEM-FEG)

\begin{tabular}{|c|c|c|c|c|c|c|}
\hline- & Test 2 (ladle) & Test 3 (ladle) & Test 4 (furnace) & $\begin{array}{l}\text { Test } 5 \text { (ladle } \\
\text { and furnace) }\end{array}$ & $\begin{array}{l}\text { Test } 6 \text { (ladle } \\
\text { and furnace) }\end{array}$ & $\begin{array}{l}\text { Test } 7 \text { (ladle } \\
\text { and furnace) }\end{array}$ \\
\hline Porosity & 2069 & 2113 & 790 & 797 & 645 & 670 \\
\hline MnS Type I or III & 114 & 98 & 150 & 38 & 108 & 113 \\
\hline MnS Type II & 55 & 45 & 7 & 18 & 57 & 45 \\
\hline MnS total & 69 & 143 & 157 & 56 & 165 & 158 \\
\hline $\mathrm{Al}_{2} \mathrm{O}_{3}$ & 78 & 320 & 3 & 420 & 623 & 405 \\
\hline
\end{tabular}

The important amount of porosity in Tests 2 and 3 show that deoxidation in the ladle alone is insufficient. So, it is necessary to kill the bath thanks to the addition of aluminum inside the furnace. Tests 4, 5, 6, and 7 highlight that this step allows us to reduce number of porosities by 3 . Nevertheless, the transfer between the furnace and the ladle causes a reoxidation of the steel. This is why an additional deoxidation has to be done in the ladle. This phenomenon is shown by the presence of $\mathrm{Al}_{2} \mathrm{O}_{3}$ in the steel in Tests 5, 6, and 7 realized with two aluminum additions. Finally, the amount of $\mathrm{Al}_{2} \mathrm{O}_{3}$ in Tests 5, 6, and 7 is more or less equivalent. So, the quantity of $\mathrm{Al}_{2} \mathrm{O}_{3}$ is independent of the weight of the aluminum added for the second deoxidation. This is why the minimum addition of Al (Test 7) is sufficient for the deoxidation in the ladle.

First, Tests 2 and 3 have been realized to show the repeatability of the experimental protocol and of the characterization method. The results shown in Table 4 being nearly similar reveals this repeatability. Deoxidation in the furnace in addition to traditional deoxidation in the ladle can really improve the oxygen treatment in steel.

The quantity of MnS Types I and III is at its lowest level with aluminum added in the furnace. MnS Type II is the worst type of inclusion (as previously seen) [8]. The total amount of sulfide is not correlated with the residual amount of sulfur (Tab. 3); however, the amount of sulfur could affect the size and morphology of the inclusions.

In Test 5 , the quantity of MnS inclusions is lower. The sulphide nucleation could be related to the amount of aluminum. So, the hypothesis is that the quantity of those particles could be equivalent to that of Tests 6 and 7, but their size is too small to be detected with the parameters chosen in the software. Because of this singularity in Test 5 , it would be interesting to analyze the sizes and morphology of the inclusions in more-exhaustive ways (which are possible thanks to the AZTEC software).

\section{CONCLUSION}

First, this study gives information about steel elaboration and particularly its deoxidation. The whole process of deoxidation used in this study was realized during two different steps in the steelmaking; in the furnace, and in the ladle. Characterizations of the obtained steel using spark spectrometer and SEM with software image analyses allow us to determine the best process of deoxidation. Spark spectrometer shows that aluminum is totally consumed in the furnace but completely integrated in the ladle. The oxygen activity consumes the aluminum in the furnace, but the rest of the oxygen activity is too low in the ladle to consume all of the aluminum. The porosity in steel is also lower with a deoxidation furnace (as the SEM analyses show). So, completing deoxidation with a furnace aluminum addition increases the quality of the steel. The content of $1 \mathrm{~kg} /$ ton is still enough in the ladle to complete deoxidation. To complete these results, measurement of the oxygen activity in liquid steel by the adapted equipment would be helpful to confirm the choice of deoxidation.

Then, the analyses of inclusion cleanliness show that the quantity of sulfur in the steel does not affect the quantity of the MnS inclusions. The mechanism of MnS nucleation is more linked to the alumina quantity than sulfur quantity. MnS morphology can evolved with the deoxidation process. A better mastery of AZTEC would allow for a finer analysis concerning the counting and inclusion classification.

Finally, the numerical method of inclusion counting makes it possible to cover a larger surface in less time than the traditional optical method (for the same surface, the numerical method is 4.6 times faster than the optical method). It also makes it possible to obtain all of the chemical compositions of all of the particles. This method also reduces the bias of the observer during the inclusion count. This avoids missing inclusions or counting porosities as inclusions. The software makes it possible to distinguish between the globular and elongated types of MnS inclusions, but this method needs to be further studied in order to draw conclusions about their respective quantities in steel. This software is very efficient and shows possible improvements in aluminum deoxidation in low-carbon steel.

\section{Acknowledgments}

This study was realized with the financial support of SAFE Metal.

\section{REFERENCES}

[1] Zhang G.-H. \& Chou K.-C. (2015). Deoxidation of molten steel by aluminum. Journal of Iron and Steel Research, International, 22(10), 905-908. doi:10.1016/S1006-706X(15)30088-1

[2] Li Y., Wan X. L., Lu W.Y., Shirzadi A.A., Isayev O., Hress 0. \&Wu K.M. (2016).Effect ofZr-Ti combined deoxidation on the microstructure and mechanical properties of high-strength low-alloy steels. Materials Science and Engineering A, 659, 179-187. doi:10.1016/j.msea.2016.02.035

[3] Golubtsov V.A., Shub L.G., Deryabin A.A. \& Usmanov R.G. (2006). Treating steel outside the furnace more efficiently. Metallurgist, 50(11-12),634-637. doi:10.1007/s11015-0060135-1 
[4] Li Z., Liu C., Sun Q. \& Jiang M. (2015). Effect of deoxidation process on distribution characteristics of inclusions in silicon steel slabs. Journal of Iron and Steel Research, International, 22(Supplement 1), 104-110. doi:10.1016/S1006-706X (15) $30147-3$

[5] Yarwood J.C., Flemings M.C. \& Elliott J.F. (1971). Inclusion formation in the Fe-O-S system. Metallurgical Transactions, 2(9), 2573-2582. doi:10.1007/BF02814897

[6] Zhang L. \& Thomas B.G. (2006). State of the art in the control of inclusions during steel ingot casting. Materials and Metallurgical Transactions, 37(5), 733-761.

[7] Ito Y., Masumitsu N. \& Matsubara K. (1981). Formation of manganese sulfide in steel. Transactions of the Iron and Steel Institute of Japan, 21(7), 477-484. doi:10.2355/ isijinternational1966.21.477
[8] Editions Techniques des Industries de la Fonderie. (2007). Méthode micrographique de détermination de la teneur en inclusions non métalliques des aciers moulés. NF EN 10247.

[9] Pokorny A. \& Pokorny J. (1998). Inclusions non métalliques dans l'acier. Techniques de l'Ingenieur, 33(M220), 1-43.

[10] Hénault E. (2006). Method of automatic characterization of inclusion population by a SEM-FEG/EDS/Image. JEOL News, $41 \mathrm{E}(1), 22-24$.

[11] Le Coze J. \& Saleil J. (2015). La propreté des aciers: une longue conquête scientifique et technologique de la sidérurgie. Matériaux et Techniques, 103(5), 1-16. https://doi. org $/ 10.1051 /$ mattech $/ 2015047$

[12] ASTM International (2005). ASTM E45-05 Standard Test Methods for Determining the Inclusion Content of Steel, 19. doi:10.1520/E0045-05E03.2 